\title{
Diffuse Cerebral Sclerosis of Schilder
}

National Cancer Institute

\section{Source}

National Cancer Institute. Diffuse Cerebral Sclerosis of Schilder. NCI Thesaurus. Code C84670.

A rare congenital demyelinating disorder affecting the central nervous system. It is characterized by a demyelinating destructive lesion affecting an entire brain lobe or hemisphere. Signs and symptoms include dementia, cortical deafness and blindness, pseudobulbar palsy, and hemiplegia. 